\title{
Energy band-gap dependence of two-photon absorption
}

\author{
Eric W. Van Stryland, M. A. Woodall, H. Vanherzeele, and M. J. Soileau \\ Center for Applied Quantum Electronics, Department of Physics, North Texas State University, Denton, Texas 76203
}

Received February 7, 1985; accepted July 26, 1985

\begin{abstract}
We present measurements of the two-photon absorption coefficients $\beta_{2}$ of 10 different semiconductors having band-gap energies between 1.4 and $3.7 \mathrm{eV}$. We find that $\beta_{2}$ varies as $E_{g}{ }^{-3}$, as predicted by theory. In addition, the absolute values of $\beta_{2}$ agree with theory, which includes the effect of nonparabolic bands, the average difference being less than $26 \%$. This agreement permits confident predictions of two-photon absorption coefficients of other materials at other wavelengths.
\end{abstract}

The ever-increasing role of semiconductors in lightwave technology has created a pressing demand for the characterization of the nonlinear-optical properties of these materials. Semiconductors are attractive as elements in nonlinear-optical devices because of their large and potentially extremely fast optical nonlinearities. A careful study of these macroscopic nonlinearities should allow one to determine the dependence of these nonlinearities on fundamental microscopic mechanical and electronic material properties (e.g., band gap, carrier lifetime, carrier effective mass). The data base formed by this information would then allow one not only to tabulate the materials that exhibit large nonlinearities but also to predict the specific material parameters that give rise to these high nonlinearities. This predictive capability is extremely important from the standpoint of searching for materials with large nonlinearities.

A study of the nonlinear-optical properties of several semiconductors is presented here, and a relationship between the two-photon absorption coefficient $\left(\beta_{2}\right)$ and other material properties is verified. Ten different materials were experimentally studied for which the incident photon energy $\hbar w$ is less than the direct band-gap energy $E_{g}$ but greater than $E_{g} / 2$, so that two-photon absorption (2PA) is allowed. ${ }^{1}$ Both 1.06and $0.53-\mu \mathrm{m}$ picosecond pulses are used in transmission experiments, similar to those used previously by Bechtel and Smith, ${ }^{2}$ on semiconductors with $E_{g}$ ranging from 1.4 to $3.7 \mathrm{eV}$. We find that $\beta_{2}$ is given by

$$
\beta_{2}=K \sqrt{E_{p}} F\left(2 \hbar w / E_{g}\right) / n^{2} E_{g}{ }^{3},
$$

where $k$ is a material-independent constant, $n$ is the linear refractive index, and $E_{p}$ (related to the Kane momentum parameter ${ }^{3}$ ) is nearly material independent for a wide variety of semiconductors. The function $F$, whose exact form depends on the assumed band structure, is a function only of the ratio of the photon energy $\hbar w$ to $E_{g}$, which determines the states that are optically coupled. The scaling given by Eq. (1) agrees with the most recent theories for $2 \mathrm{PA}^{4-6}$ and allows for predictions of 2PA coefficients for other materials at other wavelengths given minimal material parameters. For example, extension of this scaling to InSb $(300 \mathrm{~K})$ at $10.6 \mu \mathrm{m}$ predicts a $\beta_{2}$ of $6.8 \mathrm{~cm} / \mathrm{MW}$, which is in excel- lent agreement with recent experiments. Specifically, Miller et al. ${ }^{7}$ obtain a value of $8 \mathrm{~cm} / \mathrm{MW}$. Equation (1) is therefore valid over a range of 20 in $E_{g}$ from the infrared to the visible. In addition, we find that the proportionality constant $K$ as calculated by Weiler ${ }^{5}$ for nonparabolic bands agrees with our experimentally determined $K$ to within better than $26 \%$.

The picosecond-pulse-transmission experiments used to extract $\beta_{2}$ are similar to the measurements used by Bechtel and Smith. ${ }^{2}$ Further experimental details are given in Ref. 1. The absorption due to two-photongenerated free carriers was determined to be negligible; however, the induced index-of-refraction changes introduced by them were quite large. The observed defocusing can be explained entirely by the photogenerated carriers as discussed in Ref. 8 and can be used to greatly enhance the optical limiting caused by $2 \mathrm{PA} .{ }^{9}$

Table 1 lists the materials, the relevent materialdependent parameters, and the measured $\beta_{2}$ 's. The results of these measurements are summarized in the next to the last column of Table 1 . The absolute-error bars on the values of $\beta_{2}$ are estimated to be $\pm 40 \%$. We conservatively estimate the relative error bars from one sample to the next, which are important in determining the parametric dependence of $\beta_{2}$ as discussed in the following paragraphs, to be $\pm 25 \%$.

Figure 1 displays the data as a log-log plot of the experimentally determined $\beta_{2}$ 's, scaled by parameters in Eq. (1), as a function of band-gap energy. The dependence of $\beta_{2}$ on $E_{g}$ predicted from Eq. (1) would yield a line of slope -3 on such a graph. The solid line in Fig. 1 is a least-squares fit of the data to a line of slope -3 and shows the excellent agreement of the $E_{g}$ scaling given by Eq. (1). The functional dependence of $F$ used to obtain Fig. 1 is taken from Refs. 5 and 6 for parabolic bands and is given by

$$
F\left(2 \hbar w / E_{g}\right)=\left(2 \hbar w / E_{g}-1\right)^{3 / 2} /\left(2 \hbar w / E_{g}\right)^{5} .
$$

The value of $K$ in Fig. 1 obtained from this fit is 3100 , where $E_{p}$ and $E_{g}$ are in electron volts and $\beta_{2}$ is in centimeters per gigawatt. The predicted values of $\beta_{2}$ using parabolic bands and Eq. (1) are given in the last column of Table 1 . These values are on average $\sim 1.7$ times lower than predicted for parabolic bands from Ref. 5 . However, if the dependence of $F$ on $2 \hbar w / E_{g}$ as calcu- 
Table 1. Material Parameters and Two-Photon Absorption Coefficients of the Materials Studied

Two-photon Absorption: $\lambda=1.06 \mu \mathrm{m} \quad 2 \hbar \omega=2.34 \mathrm{eV}$

\begin{tabular}{|c|c|c|c|c|c|c|c|}
\hline Material & form $(a)$ & $n(\lambda)$ & $E g(e V)$ & $E_{p}^{(b)}(e V)$ & $E_{b} / E_{g}$ & $\beta_{2}^{\exp }\left(\frac{\mathrm{cm}}{\mathrm{GW}}\right)$ & $\beta_{2}^{\text {theor. }}\left(\frac{\mathrm{cm}}{\mathrm{GW}}\right)$ \\
\hline $\mathrm{ZnTe}{ }^{(c)}$ & $\mathbf{Z}$ & $2.79(c)$ & $2.26^{(i)}$ & 19.1 & $0.004^{(0)}$ & 4.5 & 0.89 \\
\hline $\mathrm{CdSe}{ }^{(c)}$ & $w$ & $2.56^{(c)}$ & $1.74^{(j)}$ & 21 & $0.007^{(i)}$ & 18 & 18.6 \\
\hline$C d \mathrm{Ce}^{(\mathrm{d})}$ & Z & $2.84^{(c)}$ & $1.44^{(c)}$ & 20.7 & $0.003^{(0)}$ & 22 & 25.1 \\
\hline $\mathrm{CdTe}^{(\mathrm{d})}$ & $\mathrm{Zp}$ & $2.84^{(c)}$ & $1.44^{c)}$ & 20.7 & $0.003^{(0)}$ & 15 & 25.1 \\
\hline $\mathrm{CdS}_{.5} \mathrm{Se}(\mathrm{c})$ & $w$ & $2.45^{(1)}$ & $1.93^{(k)}$ & 21 & $0.010^{(I)}$ & 10 & 12.1 \\
\hline $\mathrm{CdS}_{.25} \mathrm{Se} .75$ & $w$ & $2.51^{(1)}$ & $1.78^{(k)}$ & 21 & $0.008^{(I)}$ & 15 & 17.7 \\
\hline $\mathrm{GaAs}(\mathrm{e})$ & $\mathbf{Z}$ & $3.43^{(i)}$ & $1.42^{(i)}$ & 25.7 & $0.003^{(0)}$ & 23 & 19.7 \\
\hline \multicolumn{2}{|c|}{ Two-photon Absorption: } & \multicolumn{2}{|c|}{$\lambda=0.53 \mu \mathrm{m}$} & \multicolumn{2}{|c|}{$2 \hbar \omega=4.68 \mathrm{eV}$} & & \\
\hline Material & form & $\mathbf{n}(\lambda)$ & $\mathrm{Eg}(\mathrm{eV})$ & $E p(e V)$ & $E_{b} / E_{g}$ & $\beta_{2}^{\exp }\left(\frac{\mathrm{cm}}{\mathrm{Gw}}\right)$ & $\beta_{2}^{\text {theor. }}\left(\frac{\mathrm{cm}}{\mathrm{GW}}\right)$ \\
\hline $\mathrm{ZnS} \mathrm{S}^{(\mathrm{f})}$ & $Z p(c)$ & $2.40^{(i)}$ & $3.66^{(i)}$ & 20.4 & $0.010^{(i)}$ & 2.0 & 2.10 \\
\hline $\mathrm{ZnS}^{(f)}$ & $\mathrm{Zp}$ & $2.40^{(i)}$ & $3.66^{(i)}$ & 20.4 & $0.010^{(i)}$ & 3.5 & 2.10 \\
\hline $\mathrm{ZnSe}{ }^{(g)}$ & $Z p$ & $2.70^{(i)}$ & $2.67^{(i)}$ & 24.2 & $0.008^{(0)}$ & 5.5 & 4.27 \\
\hline $\mathrm{CdS}^{(\mathrm{c})}$ & $w$ & $2.60^{(i)}$ & $2.42^{(c)}$ & 21 & $0.012^{(i)}$ & 5.5 & 4.87 \\
\hline $\mathrm{ZnO} \mathrm{O}^{(\mathrm{h})}$ & $w$ & $2.05^{(i)}$ & $3.20^{(n)}$ & 21 & $0.020^{(i)}$ & 5.0 & 4.77 \\
\hline
\end{tabular}

Note: $E_{b}$ is the exciton-binding energy.

${ }^{(a)} \mathrm{z}$, zinc blende; w, wurtzite; p, polycrystalline. ${ }^{(b)}$ Ref. 10. ${ }^{(c)}$ Ref. 11. ${ }^{(d)}$ Ref. 12. ${ }^{(e)}$ Ref. 13. ${ }^{(f)}$ Ref. $14 .{ }^{(g)}$ Ref. $15 . \quad{ }^{(h)}$ Ref. $16 . \quad{ }^{(i)}$ Ref. 17. (j) Ref. 18. (k) Ref. 19. (l) Ref. 20. ${ }^{(n)}$ Ref. 21. ${ }^{(o)}$ Ref. 5.

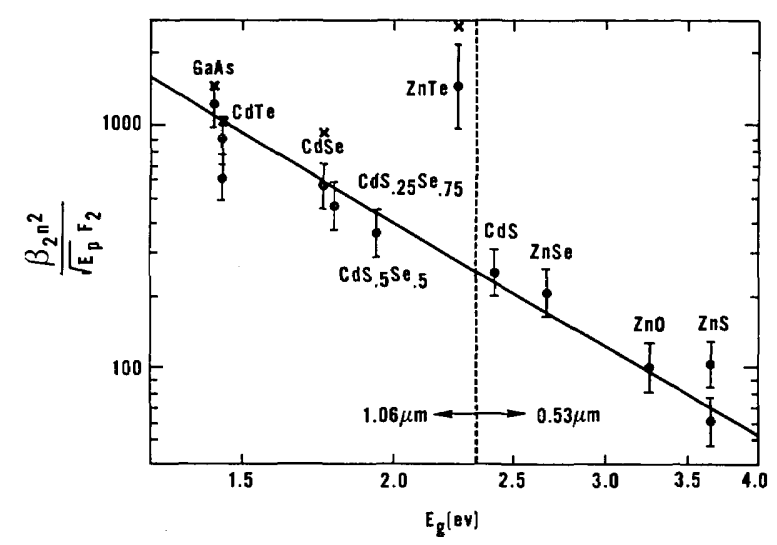

Fig. 1. A log-log plot of the scaled 2PA coefficient versus energy gap assuming parabolic band structure. The solid line is a least-squares fit of the data to a line of slope -3 (omitting $\mathrm{ZnTe}$ ). The X's shown for GaAs, CdTe, CdSe, and ZnTe are data from Ref. 11. Data to the left of the vertical dotted line were taken with $1-\mu \mathrm{m}$ light; those to the right, with $0.5-\mu \mathrm{m}$ light.

lated for nonparabolic bands in Ref. 5 is used in Eq. (1), the absolute values agree considerably better on average, although the overall fit is not so good. We find that the data of Table 1 are $26 \%$ lower on average than predicted for nonparabolic bands with the spin-orbit splitting $\Delta \ll E_{g}$ and $3 \%$ lower than predicted for $\Delta \gg$ $E_{g} .5$ For the materials tested $\Delta$ lies between these two limiting cases.

The $\beta_{2}$ for $\mathrm{ZnTe}$ is considerably larger than expected based on the empirical fit of Eq. (1) as seen in Fig. 1. The data of Fig. 1 are replotted in Fig. 2 to show the variation of $F_{2}$ with $2 \hbar w / E_{g}$. This graph is similar to graphs given in Refs. 4-6. Figure 2 shows that for $\mathrm{ZnTe}$ two photons couple states that are only $3.5 \%$ above the energy gap where $F_{2}$ (primarily a density-of-states factor $\left.^{6}\right)$ is small, and thus $\beta_{2}$ is expected to be small. Near the gap, however, is where the effects of excitons are expected to be greatest. If exciton enhancement, as calculated by Lee and Fan, ,,22 is included, most of the discrepancy of $\beta_{2}$ for $\mathrm{ZnTe}$ from the values of $\beta_{2}$ for the other materials, as shown in Figs. 1 and 2, is removed. However, the overall fit of the data to theory is considerably worse than displayed in Figs. 1 and 2. This may be attributed to our lack of accuracy in determining the exciton enhancement of $\beta_{2}$, which depends, for example, on the exciton-binding energy (see Table 1). ${ }^{5,22} \mathrm{Al}$ though we have only one data point coupling states so near the band gap, the data of Bechtel and Smith ${ }^{2}$ are in excellent agreement where there is overlap (see the $X$ 's in Fig. 1). In particular, Bechtel and Smith also obtained a large $\beta_{2}$ for $\mathrm{ZnTe}$. 


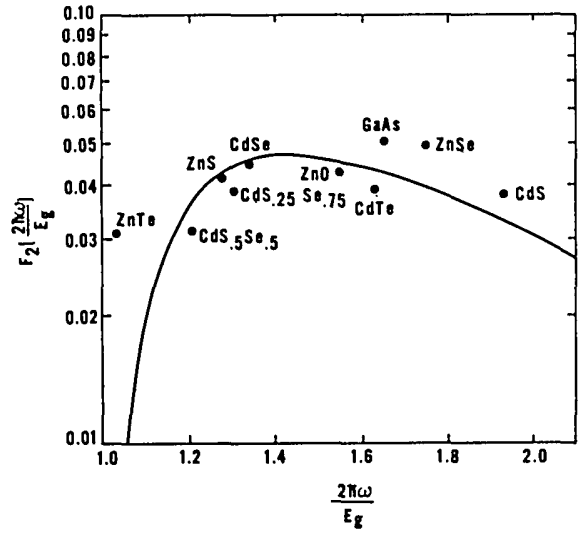

Fig. 2. The function $F_{2}$ plotted versus $2 \hbar w / E_{g}$ using $K=$ 3100 in Eq. (1). ( $E_{p}$ and $E_{g}$ are in electron volts, and $\beta_{2}$ is in centimeters per gigawatt.)

If we attempt to extend this theory into the UV to wide-gap ionic solids (for which the band structure is clearly different from the semiconductors studied) we find that the predictions are in general considerably lower than the experiments; however, a general trend can be found. If the coupled states are well above the gap the deviations are relatively small, of the order of a factor of 4 or 5 . As the coupled states get close to the gap, however, the deviation increases rapidly. For example, in RbI at $266 \mathrm{~nm}$, where $2 \hbar w / E_{g}=1.47$, the experimentally measured $2 \mathrm{PA}$ coefficient of $2.49 \mathrm{~cm} / \mathrm{GW}$ (Ref. 23) is $\sim 3.7$ times larger than predicted by Eq. (1). At a wavelength of $355 \mathrm{~nm}$, where $2 \hbar w / E_{g}=1.10$, however, the measured $2 \mathrm{PA}$ coefficient of $5.08 \mathrm{~cm} / \mathrm{GW}$ (Ref. 23) is 14 times larger than predicted. This trend is maintained for the limited data available. ${ }^{23}$ Such large correction factors may be accounted for by considering color centers as excitons with large binding energies.

The difference in $\beta_{2}$ values for the two $\mathrm{ZnS}$ samples may be explained by the contribution of defects to $\beta_{2}$. The $\mathrm{ZnS}(y)$ sample is a chemical-vapor-depositiongrown sample that has a yellow appearance caused by crystal lattice imperfections that can be annealed out by a special heat-treatment process. ${ }^{14}$ The $\mathrm{ZnS}(c)$ is the same starting material that has undergone this heat treatment. It appears water clear, and its linear transmission cutoff is shifted into the UV. The indication from the data presented here is that not only has the linear absorption in the visible and near-UV been reduced but the two-photon absorption at $0.5 \mu \mathrm{m}$ has also been reduced by this heat treatment.

The fact that the scaling of Eq. (1) fits the data so well for a wide variety of semiconductors implies that all the important materials parameters have been included in the theory and that other contributions to $\beta_{2}$ (e.g., higher bands) cause small effects. Even more remarkable is the fact that although the theories were developed for zinc-blende materials they also experimentally fit the wurtzite data. Only when two photons couple states very close to the gap does a significant departure occur.

The material parameter dependence found for this wide variety of semiconductors allows us to predict, with reasonable confidence, the $2 \mathrm{PA}$ coefficient of other materials at other wavelengths. This includes, for example, mixed ternary compounds. Thus the $2 \mathrm{PA}$ at a particular wavelength can be tailored for a specific application.

We wish to express our thanks to B. S. Wherrett, with whom we had several useful and enlightening discussions. This research was supported with funds from the National Science Foundation (ECS \#8310625), the U.S. Office of Naval Research, and the Defense Advanced Research Projects Agency.

\section{References}

1. E. W. Van Stryland, H. Vanherzeele, M. A. Woodall, M. J. Soileau, A. L. Smirl, S. Guha, and T. F. Boggess, Opt. Eng. 24, 613 (1985).

2. J. H. Bechtel and W. L. Smith, Phys. Rev. B 13, 3515 (1976).

3. $E_{p}=2 P^{2} m / \hbar^{2}$, where $P$ is the Kane momentum parameter and $m$ is the electron mass; E. O. Kane, J. Chem. Phys. Solids 1, 249 (1957).

4. C. R. Pidgeon, B. S. Wherrett, A. M. Johnston, J. Dempsey, and A. Miller, Phys. Rev. Lett. 42, 1785 (1979).

5. M. Weiler, Solid State Commun. 39, 937 (1981).

6. B. S. Wherrett, J. Opt. Soc. Am. B 1, 67 (1984).

7. A. Miller, A. Johnston, J. Dempsey, J. Smith, C. R. Pidgeon, and G. D. Holah, J. Phys. 12, 4839 (1979).

8. S. Guha, E. W. Van Stryland, and M. J. Soileau, Opt. Lett. 10, 285 (1985).

9. T. F. Boggess, A. L. Smirl, S. C. Moss, I. W. Boyd, and E. W. Van Stryland, IEEE J. Quantum Electron. (to be published).

10. Values taken from E. O. Kane, in Narrow Gap Semiconductors-Physics and Applications, W. Zawadzki, ed. (Springer-Verlag, New York, 1980), p. 13; for values not listed in this reference the value of $21 \mathrm{eV}$ was assumed.

11. Cleveland Crystals, P.O. Box 17157, Euclid, Ohio 44117.

12. II-VI, Inc., Saxonburg Boulevard, Saxonburg, Pa. 16056.

13. Morgan Semiconductors, 2623 National Circle, Garland, Tex. 75041.

14. CVD Inc., 35 Industrial Parkway, Woburn, Mass. 01801.

15. Raytheon Company, Missile Systems Division, Hartwell Road, Bedford, Mass. 01730.

16. Atomergic Chemetals, 100 Fairchild Avenue, Plainview, N.Y. 11803.

17. K. H. Hellwege, ed., Landolt-Bornstein Numerical Data and Functional Relationships in Science and Technology, Vol. 17, Subvols. (a) and (b), Semiconductors (Springer-Verlag, New York, 1982).

18. M. Neuberger, "II-VI semiconducting compound tables," (Hughes Aircraft Company, Culver City, Calif., 1969).

19. Y. S. Park and D. C. Reynolds; Phys. Rev. 132, 2450 (1963).

20. These values were obtained by linear extrapolation as a function of composition between the known values for CdS and CdSe. Y. S. Park and D. C. Reynolds, Phys. Rev. 132, 2450 (1963).

21. R. H. Bube, Photoconductivity of Solids (Wiley, New York, 1960).

22. C. C. Lee and H. Y. Fan, Phys. Rev. B 9, 3502 (1974).

23. P. Liu, W. L. Smith, H. Lotem, J. H. Bechtel, and N. Bloembergen, Phys. Rev. B 17, 4620 (1978). 